\title{
Classification of Parasomnias
}

\author{
Louis Kazaglis $^{1,2}$ • Michel A. Cramer Bornemann ${ }^{3,4}$
}

Published online: 30 March 2016

(C) Springer International Publishing AG 2016

\begin{abstract}
Parasomnias, or undesirable events accompanying sleep, encompass a broad spectrum of behaviors and experiences. Classification of parasomnias has undergone a significant transformation in the International Classification of Sleep Disorders Third Edition. Underlying this new classification is the understanding that Wake, NREM sleep, and REM sleep are not mutually exclusive states. Recent advances in neuroscience support the State Dissociation paradigm which would allow for an unstable admixture of components of these states to coexist. Parasomnias are the clinical manifestation of this state instability. This article briefly reviews the characteristics, demographics, and pathophysiology of the core parasomnias and associated disorders, with emphasis on disorders of arousal and diagnosis and prognostic significance of REM sleep behavior disorder.
\end{abstract}

This article is part of the Topical Collection on Parasomnias

Michel A. Cramer Bornemann

Miche19626@yahoo.com

Louis Kazaglis

Louis.Kazaglis@gmail.com

1 Minnesota Regional Sleep Disorders Center, Hennepin County Medical Center, 701 Park Avenue, G8.320, Minneapolis, MN 55415, USA

2 Department of Medicine, School of Medicine, University of Minnesota, Twin Cities, MN, USA

3 Olmsted Medical Center, Rochester Northwest, 5067 55th Street NW, Rochester, MN 55901, USA

4 Sleep Medicine Fellowship, Minnesota Regional Sleep Disorders Center, Hennepin County Medical Center, Minneapolis, MN, USA
Keywords Confusional arousals · Disorders of arousal · Parasomnia $\cdot$ REM sleep behavior disorder $\cdot$ Sleep terrors . State dissociation

\section{Introduction}

Parasomnias, or undesirable events accompanying sleep, encompass a broad spectrum of behaviors and experiences. Classification of parasomnias has undergone a significant transformation in the most recent iteration of the International Classification of Sleep Disorders (ICSD). Parasomnias can be best understood using the State Dissociation paradigm. This article briefly reviews the characteristics, demographics, and pathophysiology of the core parasomnias and associated disorders, with emphasis on Disorders of Arousal and REM sleep behavior disorder.

\section{Background}

The word parasomnia derives from the Greek prefix para, meaning alongside of, combined with the Latin noun somnus for sleep, to account for the dictionary definition of events that accompany sleep. In the clinical setting, the term thereby relates to undesirable events that accompany sleep. Accordingly, parasomnias encompass a broad spectrum including dreaming, misperceptions, dysphoric emotions, abnormal sleep-related movements as well as behaviors, and dysregulated autonomic nervous system functioning. Parasomnias become clinical disorders as they may result in sleep fragmentation, adverse health effects, troublesome psychosocial effects, and even injuries. The latter may have potential forensics implications. Consequently, addressing safety is often an initial goal as the clinical impact may involve more 
than just the patient, depending upon the complexity and duration of the sleep-related behaviors.

Parasomnias have long been defined in the International Classification of Sleep Disorders (ICSD) as undesirable physical events or experiences that occur during entry into sleep, within sleep, or during arousals from sleep. Furthermore, parasomnias may occur during non-rapid eye movement sleep (NREM), rapid eye movement sleep (REM), and during transitions to and from sleep. However, such a definition, though technically correct, is overly simplistic, open for misinterpretation, and vulnerable for misapplication. In an age in which an inquisitive mind may almost immediately satiate their thirst for knowledge with the stroke of a few keypads when interacting with an internet search engine, healthcare providers must remain vigilant concerning web-based information that are often uncritically accepted by patients. The general public and popular media continue to be captivated by the belief that the unconscious mind, in either a state of hypnosis or sleep, may reveal itself in words, mental images, or behaviors though its meaning is kept at a distance from the conscious mind through a barrier of repression. Various avenues for the interpretation into dreams have evolved based upon the premise that behaviors and/or experiences which arise from the platform of sleep are the result of a breakdown of psychic censorship and may thereby provide insight into the machinations of the unconscious mind. Such "Dream Theory," including that developed by Sigmund Freud, which may masquerade in subtle forms including that as "wish fulfillment," especially when applied to parasomnias has been supplanted by modern neuroscientific constructs that are driven by rigorous methodology that require hypotheses that are both testable and verifiable. As such, the human condition is now understood to be comprised of three essential states: Wake, NREM sleep, and REM sleep. It is the dynamic interplay of these three states which can account for previously difficult to explain human experiences. Nevertheless, the pseudoscience of certain aspects of "Dream Theory" continues to hold sway over the general public as supported by the everlasting success of dream interpretation manuals and the belief in the metaphorical significance of dream symbolism. Particularly in cases with legal implications, it is important to recognize when older, now discredited, paradigms for understanding human behavior remain in play.

Recent advances in neuroscience coupled with refined neurodiagnostic imaging modalities now reveal that the three states are modulated by a host of physiologic influences including the degree of aminergic and cholinergic neurochemical bias, CNS activation, and the degree of endogenous vs. exogenous input. Directly influenced by regularly recurring physiologic conditions including homeostatic drive and circadian rhythmicity, the process of state declaration is most often maintained in a stable and predictable fashion throughout a 24-h period. However, as the components of sleep frequently oscillate, it is not unexpected that the brain with its estimated 85-100 billion neurons [1] may experience brief episodic electrochemical switching errors. In such situations, sleep and wake may be abruptly rendered into a state that is not yet fully declared and in a temporary unstable state of dissociation. Thus, sleep and wake, as well as its associated consciousness and unconsciousness, are not dichotomous states as they occur on a spectrum and are evanescent [2].

The most current version of the International Classification of Sleep Disorders (ICSD third Edition) now recognizes State Dissociation as the paradigm for parasomnias [3・・]. Previously, wakefulness, non-rapid eye movement sleep (NREM), and rapid eye movement sleep (REM) were thought to be the three states that were essentially mutually exclusive. Recent research has shown that combinations of one or more of these states is possible and may result in unusual unstable states that potentially manifest as parasomnias or as altered levels of awareness [4-8]. Appreciation for the State Dissociation paradigm facilitates a neurophysiologic mechanistic approach to understand parasomnias as well as a refinement of its classifications. NREM-related parasomnias are an admixture of wakefulness and NREM sleep and include confusional arousals, somnambulism, and sleep terrors. Here, the prefrontal cortex is considered to be "offline" with subsequent severe impairment, or absence, of higher executive cognitive functioning while potential for motor capacity may be retained $[9,10]$. REM-related parasomnias are an admixture of the elements of REM sleep together with wakefulness. In contrast with NREM-related parasomnias, these individuals often recollect emotionally dysphoric dreams. Aside from REM sleep behavior disorder (RBD), the admixture of REM sleep and wakefulness explains the experiences of sleep paralysis, cataplexy, and hypnagogic hallucinations which are seen in narcolepsy. All three states may be present in the same individual as overlap disorders. As parasomnias arise from the platform of sleep, behaviors attributed to this condition are performed without awareness.

One should remain mindful that there are several abnormal sleep-related movements that do not adhere to the State Dissociation paradigm. These are important clinical conditions worthy of separate classification which have been placed under the heading of "Sleep Related Movement Disorders." This heading is most notable for restless legs syndrome (RLS), otherwise referred to as Willis Ekbom Disease (WED). Abnormal sleep-related movements encompass a broad range of simple movements including bruxism as well as myoclonic, rhythmic, rocking, cramping, and dyskinetic movements or tremors, which are not characteristically associated with dream mentation.

The current iteration of the ICSD third edition recognizes 10 core categories of parasomnias (Table 1). Only one of the core categories, rapid eye movement (REM) sleep behavior disorder (RBD), requires video polysomnographic 
Table 1 (see REF. 3) Classification of parasomnias

NREM-related parasomnias
Disorders of arousal
Confusional arousals
Sleepwalking
Sleep terrors
Sleep-related eating disorder
REM-related parasomnias
REM sleep behavior disorder
Recurrent isolated sleep paralysis
Nightmare disorder
Other parasomnias
Exploding head syndrome
Sleep-related hallucinations
Sleep enuresis
Parasomnias due to medical disorder
Parasomnias due to medication or substance
Parasomnia, unspecified
Isolated symptom/normal variant
Sleep talking (somniloquy)

documentation as one of the essential diagnostic criteria. However, for most of the other parasomnias, polysomnographic monitoring can provide corroborative documentation in support of the clinical diagnosis. The ICSD third edition is largely based upon peer-reviewed consensus-driven clinicalbased evidence in conjunction with up-to-date pathophysiologic developments in somnology and neuroscience. Any classification system of disorders in medicine would appear to play several key roles. First, it serves as a guide for healthcare providers in the identification of specific disease states which in turn would drive focused therapeutic strategies resulting in effective clinical outcomes. One should also recognize that formal recognition of a disease state by a medical professional society also may influence future research agendas and mobilize funding subsequently improving personal and public health. Lastly, particularly, in the realm of parasomnias with its potential for sleep-related violence, formal recognition of certain conditions, including sleep-related abnormal sexual behavior (sexsomnia), can now be considered in the development of legal arguments and admissible in a court of law as these uphold the Daubert Standard in criminal cases where a sleep disorder or condition may be involved [11].

\section{NREM-Related Parasomnias}

The NREM-related parasomnias arise from an admixture state of wakefulness and NREM sleep and are termed Disorders of Arousal. Confusional arousals, sleepwalking, and sleep terrors are the primary disorders of arousal. Sleep-related eating disorder (SRED) is a subset of sleepwalking while sexsomnia is a unique form of confusional arousal. Recent electromagnetic tomographic studies have demonstrated early localized arousal-related activation of the cingulate motor area preceding sleepwalking further support the State Dissociation paradigm [12].

The NREM-related parasomnias are defined by incomplete awakening, unresponsiveness, minimal cognition, and nearto-complete amnesia. They most commonly arise associated with NREM stage 3 sleep and tend to occur during the first third of the night, but are not restricted by either boundary [13]. The differential diagnosis for NREM-related parasomnias includes sleep-disordered breathing, REMrelated parasomnias, nocturnal seizures, and psychogenic dissociative disorders. Formal diagnosis of NREM-related parasomnias is typically made by clinical evaluation; however, polysomnographic evaluation is vital in atypical cases due to the $40 \%$ incidence of alternative diagnosis or precipitant [14].

\section{Confusional Arousals}

Confusional arousals present as movements and/or vocalizations confined to the bed or sleep location. The affected individual may display varying levels of confusion or sleepdrunkenness, with lesser degrees of agitation or distress. Like the other primary disorders of arousal, they are more common in children than adults, with a lifetime prevalence of $18.5 \%$ and an adult prevalence of only $2.9-4.2 \%$ [15-17]. Unsurprisingly, shift-workers have a two- to threefold increased incidence of confusional arousals, possibly related to circadian misalignment of sleep or insufficient sleep intake [18].

Sleep-related abnormal sexual behaviors, popularly referred to as sexsomnia, are thought to be a variant of confusional arousals, although they may overlap with somnambulism when the sleep surface is vacated [3••]. Inappropriate sexualized behaviors that arise from the platform of sleep encompass a broad range of behaviors from profane vocalizations, inappropriate touch/fondling, masturbation, oral sex, to sexual intercourse. These behaviors are without conscious awareness and are frequently without dream mentation. They have broad clinical, social, and legal implications [19•]. It is thought to be fairly common, with a lifetime and annual prevalence of 7.1 and $2.7 \%$, respectively [15].

\section{Somnambulism (Sleepwalking)}

Despite the misnomer, sleepwalking is a relative amnestic state not confined to simple ambulation and may include vocalization, manipulation of household objects, and even operation of motor vehicles. It can be associated with profound 
anesthesia; in one study of patients with violent parasomnias, almost $80 \%$ experienced anesthesia during violent and selfinjurious behavior without complete awakening [20]. Sleepwalking frequently recurs; in one study, $22 \%$ of sleepwalkers had daily episodes, and $44 \%$ had at least weekly episodes [21]. Sleepwalking displays an even higher childhood preponderance compared to confusional arousals, with lifetime prevalence of $22 \%$ compared to adult prevalence of $2 \%[15,17]$. Furthermore, early onset portended higher frequency of violent behaviors. Median age at sleepwalking onset is $9-10$ years, although in one series, a third of adult patients with injurious sleepwalking developed sleepwalking after the age of 16 [21, 22].

Family history of sleepwalking was reported in $57 \%$ of sleepwalkers, and the prevalence of sleepwalking was $47 \%$ among children with one parent who had a history of sleepwalking, consistent with prior studies suggesting a genetic inheritance [21-23]. Sleepwalkers may be more sensitive to sleep deprivation; resting cerebral blood flow was decreased in sleepwalkers compared to controls following a night of sleep deprivation [24]. They also display alterations in sleep microarchitecture and more frequent NREM stage 3 arousals. Evaluations of slow-wave activity and oscillation density in sleepwalkers have demonstrated differences between behavior-associated arousals and benign arousals $[25,26]$.

\section{Sleep Terrors}

As the name implies, pavor nocturnus, or sleep terrors appear externally as terrifying experiences. Sleep terrors and sleepwalking likely exist on a spectrum, differentiated by the nature of vocalization and degree of agitation. Sleep terrors consist of screaming, extreme agitation, and prominent and sometimes violent motor activity. Despite outward appearances, and in contrast to nightmare disorder, there is no dream recall, and they are essentially amnestic for the event. Perception of the environment is minimal, and attempts to abruptly terminate the episode may provoke a vigorous unintended oppositional response.

As with other disorders of arousal, sleep terrors are more common in childhood but not rare in adulthood, with a lifetime and adulthood prevalence of 10 and $2 \%$, respectively $[15,17]$. Sleep terrors typically occur at a much younger age, with peak at $11 / 2$ years of age. There appears to be a link to sleepwalking, one study showed that approximately one third of children who had sleep terrors developed sleepwalking later in life and that parental sleepwalking predicted higher rates of childhood sleep terrors [22].

\section{Sleep-Related Eating Disorder}

SRED is a type of nocturnal eating characterized by consumption of food and non-food items, not due to hunger stimulus, occurring during the typical sleep period, often without full conscious awareness and is usually not associated with other eating disorders. It is clinically distinct from night-eating syndrome which presents as recurrent consumption of large amounts of calories (in excess of $25 \%$ of daily caloric intake) after the typical evening meal with full awareness and association and is associated with other eating disorders [27]. SRED has been associated with the sedative-hypnotic medications Zolpidem and Zaleplon and the atypical antipsychotics Quetiapine and Olanzapine, among others [28-32].

At this time, it is unclear whether SRED is a disorder of arousal, a non-motor variant of RLS, an overlap syndrome between RLS and disorders of arousal, or a heterogeneous collection of disorders with similar phenotypes. SRED shares many characteristics with other disorders of arousal, including frequent amnesia, lack of awareness as evidenced by reports of consumption of non-food items, and increased NREM stage 3 arousals [15, 33]. Notably absent are the typical childhood predominance and gender equality. Supporting an association with RLS, SRED was present in $36 \%$ of RLS patients in one series [34]. Furthermore patients with RLS and SRED experienced improvement in SRED on dopamine agonist therapy and had more frequent SRED on sedative hypnotic therapy. Whether or not the relationship is due to decreased arousals from effectively treated RLS, manifestation of sedative-hypnotics medication side effects in patients with NES causing amnestic SRED or demonstration of a nonmotor RLS phenomena waits to be seen [28, 30, 34, 35].

\section{REM-Related Parasomnias}

REM-related parasomnias offer perhaps the best clinical examples of state dissociation sleep paralysis, hypnagogic/ hypnopompic hallucinations, and cataplexy had been difficult to explain human experiences but now is regarded as the manifestation of fascinating experiments in nature in which components of REM sleep mix and temporarily coexists with wakefulness often with vividly memorable oppressive apprehensiveness. Sleep paralysis is the most common example of the state dissociation of REM sleep and wakefulness. In this condition, the muscle atonia associated with REM sleep has persisted into wakefulness and is often associated with a dysphoric emotional awareness which may be accompanied by hypnopompic hallucinations.

\section{Recurrent Isolated Sleep Paralysis}

Most cases of sleep paralysis are isolated and sporadic, with $7 \%$ of the general population reporting at least one episode of sleep paralysis [36]. However, the condition can occur frequently, as in recurrent isolated sleep paralysis (RISP). RISP is described as repeated episodes of sleep paralysis causing 
significant somniphobia or fear of initiating sleep. Despite the description as isolated, episodes of sleep paralysis are commonly accompanied by hallucinatory experiences. RISP has usually been reported in a familial pattern [37].

\section{Nightmare Disorder}

Nightmares, or dysphoric threatening dreams associated with prominent recall, are thought to predominantly arise from REM sleep. The prevalence of nightmares is fairly high [15] often persisting into adulthood. Nightmare disorder is defined as recurrent nightmares associated with awakening dysphoria, and accompanied by clinically significant sleep or daytime dysfunction, such as anxiety, somniphobia, cognitive impairment, daytime sleepiness, or fatigue. Nightmare disorder is fairly rare in children outside of those with severe psychosocial stressors; however, the prevalence in adults may reach $2-$ $6 \%$ depending on estimates used [38]. The frequency is even higher in certain psychiatric subpopulations, including patients with anxiety disorders, borderline personality disorder, post-traumatic stress disorder, or psychogenic dissociative disorders.

\section{REM Sleep Behavior Disorder}

REM sleep behavior disorder (RBD) is defined as repeated episodes of vocalizations or complex behaviors during REM sleep associated with REM sleep without atonia (RWA), as defined by formal criteria established for monitoring abnormal increases in phasic or tonic muscle tone of surface EMG electrodes during REM sleep [39]. In contrast to NREM-related parasomnias, RBD often occurs with dreams that are of an emotionally dysphoric nature. Symptomatic RBD typically occurs in males starting in the sixth decade of life [40]. It is frequently described as an early precursor of neurodegenerative disorders, particularly the alpha-synucleinopathies including Parkinson's disease, dementia with Lewy Bodies, and multiple systems atrophy. In one recent series, over $80 \%$ of patients with idiopathic RBD subsequently developed a neurodegenerative disorder after a mean interval of 14 years [41]. Despite the association with neurodegenerative disorders, $\mathrm{RBD}$ is most commonly seen secondary to antidepressant medication use, typically selective serotonin reuptake inhibitor (SSRIs) or tricyclic antidepressants (TCAs), with an annual prevalence approaching $4 \%$ in psychiatric patients [42, 43]. In patients with medication-associated RBD, there is increasing gender parity and a trend towards younger patients [44, 45].

RBD requires formal diagnosis with video polysomnography in order to confirm RWA and to differentiate it from the shared features with other disorders such as NREM-related parasomnias, seizures, or nocturnal panic attacks. Testing is typically performed with augmented EMG leads on bilateral flexor digitorum superficialis muscles, as RWA classification based on quantification of flexor digitorum phasic activity has demonstrated the best accuracy [46, 47, 48•]. Evaluation of RWA requires painstaking review of 3-s micro-epochs and can be somewhat user-dependent; recent advances in standardized quantification and automated analysis will allow for more uniform evaluation and diagnosis [49-51].

Due to absence of widespread awareness of RBD, specific questioning for dream enactment behavior is crucial during clinical evaluation [40]. As polysomnography may be occasionally performed without formal sleep clinician evaluation in certain contexts, RWA may be seen during PSG without confirmatory history of dream enactment behavior. RWA is also commonly encountered in antidepressant-treated patients not meeting criteria for $\mathrm{RBD}$ diagnosis $[52 \bullet, 53]$. In these cases, clinical correlation is required, as the significance of isolated RWA remains yet to be determined

\section{Other Parasomnias}

Several other parasomnias have been described in the literature, but cannot be classified distinctly into a NREM or REMrelated parasomnia. The pathophysiology of sleep enuresis does not appear to adhere to the State Dissociation paradigm but does fit the historical definition of parasomnias and remains a very important disorder in pediatrics. Sleep enuresis, or chronic involuntary nocturnal voiding, is a combination of two subtypes, primary and secondary. Several different etiologies have been proposed, but the two subtypes are distinct clinical entities. This disorder is fairly uncommon outside of childhood. Sleep-related hallucinations (or hypnagogic or hypnopompic hallucinations) are most frequently a REMrelated phenomenon; however, drowsy-wake states may produce similar events.

Somniloquy, or sleep talking, is not actually considered a disorder according to the ICSD third edition; rather it is considered as a normal variant or isolated symptom. It is relatively common, with a lifetime prevalence of $69 \%$ [15] and is associated with disorders of arousal and REM sleep behavior disorder. Somniloquy may occasionally be unintelligible or utterances and should be differentiated from catathrenia (or sleeprelated prolonged expiratory groaning - now categorized under sleep-related breathing disorders) or other mimics of disorders of arousal.

\section{Exploding Head Syndrome}

Exploding head syndrome (EHS) is a rare benign condition best described by its other name, episodic cranial sensory shock. Sufferers report paroxysms of sudden loud noise or flashes of light experienced in the head, without cephalalgia, occurring during sleep-wake or wake-sleep transition [54]. 
Polysomnographic recording of these episodes have demonstrated occurrences during wake, as well as NREM stages 1 and 2 sleep [55]. EHS has not been formally classified as a NREM-related parasomnia likely due to differences with disorders of arousal in terms of demographics and associated factors. Numerous etiologies have been postulated, including delayed deactivation of the brainstem reticular activating system on transition to sleep, middle ear dysfunction, or sensory variant of hypnic jerks $[55,56]$. The benign nature of EHS likely precludes future research on the disorder.

\section{Secondary Parasomnias}

Secondary parasomnias also deserve consideration, particularly given the prevalence of their underlying primary conditions and potential multiplicative implications. Theoretically, any disorder that can increase sleep pressure or cause sleep fragmentation has the potential to trigger various parasomnias. Obstructive sleep apnea (OSA) has been associated with REM-related dream enactment behavior. In this condition, known as pseudo-RBD, arousals from OSA events may trigger REM-related dream enactment behavior. This typically resolves with effective treatment of OSA with positive airway pressure delivery [57].

Sleep-related abnormal sexual behaviors and other disorders of arousal may also be precipitated by OSA-triggered arousals, with potentially disastrous consequences [58]. Similarly, sleep deprivation has been associated with disorders of arousal. In clinical settings, sleep deprivation may be used in conjunction with forced arousals to assist in the diagnosis of disorders of arousal [59]. As noted above, increased frequency of disorders of arousal have also been associated with shift work and circadian misalignment $[18,60]$.

Medications have been also associated with a number of parasomnias. Zolpidem has been most commonly cited in the literature $[29,61]$, but is not alone as other benzodiazepines and benzodiazepine receptor agonists have also been implicated [34]. Nonetheless, Zolpidem is frequently associated with a number of complex sleep-related behaviors, including sleepwalking and sleep-related eating, with a recent study putting the adult incidence at $25 \%$ [62]. It is unclear if the proportion of medication-related parasomnias linked to Zolpidem is due to its high market share or to possible uniquely distinct pharmacokinetic properties not shared by other sedative-hypnotic agents. Lastly, the new class of Orexin-antagonist medications formulated for insomnia, including first-to-market Suvorexant, may carry a theoretical risk of inducing Narcolepsyassociated parasomnias such as RBD and cataplexy due to its manipulation of the Orexin system, although the true incidence of side effects in a clinical practice have yet to be defined [63].

\section{Conclusions}

The ICSD third edition recognizes 10 core categories of parasomnias. The State Dissociation paradigm allows workable conceptualization of parasomnias as changing unstable oscillatory admixtures of Wake, NREM sleep, and REM sleep. As our understanding of the neurophysiologic systems accounting for parasomnias deepens, we continue to refine the classification of these disorders. This classification allows us to identify disease states which drive therapeutic strategies for effective outcomes, define appropriate avenues for future research and public education, and guide the legal system on complex and oft-confused disorders to help determine appropriate standards [64]. Further research will hopefully continue to deepen our understanding of disorders of arousals and RBD and associated conditions with a focus on neuroprotective therapies, clarify the neurophysiologic mechanisms linking pharmacology with untoward parasomnias, and allow us to better define these behaviors across the spectrum of human existence.

Acknowledgments We would like to acknowledge Dr. Mark Mahowald for his contributions in the review and preparation of this manuscript.

\section{Compliance with Ethical standards}

Conflict of Interest Louis Kazaglis and Michel A. Cramer Bornemann declare that they have no conflicts of interest

Human and Animal Rights and Informed Consent This article does not contain any studies with human or animal subjects performed by any of the authors.

\section{References}

Paper of particular interest, published recently, have been highlighted as:

- Of importance

•. Of major importance

1. Azevedo FA, Carvalho LR, Grinberg LT, Farfel JM, Ferretti RE, Leite RE, et al. Equal numbers of neuronal and nonneuronal cells make the human brain an isometrically scaled-up primate brain. $\mathrm{J}$ Comp Neurol. 2009;513(5):532-41. doi:10.1002/cne.21974.

2. Hobson JA. REM sleep and dreaming: towards a theory of protoconsciousness. Nat Rev Neurosci. 2009;10(11):803-13. doi: 10.1038/nrn2716.

3.• International Classification of Sleep Disorders. 3rd ed. Darien: American Academy of Sleep Medicine; 2014. The newest classification of sleep disorders from the American Academy of Sleep Medicine advances the current classification of parasomnias under the State Dissociation paradigm.

4. Mahowald MW, Schenck CH. Dissociated states of wakefulness and sleep. Neurology. 1992;42(7 Suppl 6):44-51. discussion 2. 
5. Mahowald MW, Schenck CH. Dissociated states of wakefulness and sleep. In: Lydic R, Baghdoyan HA, editors. Handbook of behavioral state control: cellular and molecular mechanisms. Boca Raton: CRC Press; 1999. p. 143-58.

6. Nobili L, De Gennaro L, Proserpio P, Moroni F, Sarasso S, Pigorini A, et al. Local aspects of sleep: observations from intracerebral recordings in humans. Prog Brain Res. 2012;199:219-32. doi:10. 1016/b978-0-444-59427-3.00013-7.

7. Nobili L, Ferrara M, Moroni F, De Gennaro L, Russo GL, Campus $\mathrm{C}$, et al. Dissociated wake-like and sleep-like electro-cortical activity during sleep. NeuroImage. 2011;58(2):612-9. doi:10.1016/j. neuroimage.2011.06.032.

8. Peter-Derex L, Magnin M, Bastuji H. Heterogeneity of arousals in human sleep: a stereo-electroencephalographic study. NeuroImage. 2015;123:229-44. doi:10.1016/j.neuroimage.2015.07.057.

9. Bassetti C, Vella S, Donati F, Wielepp P, Weder B. SPECT during sleepwalking. Lancet (London, England). 2000;356(9228):484-5. doi:10.1016/s0140-6736(00)02561-7.

10. Hobson JA. Normal and abnormal states of consciousness. In: Velmans M, Schneider S, editors. The Blackwell companion to consciousness. Malden: Blackwell; 2007. p. 101-13.

11. Cramer Bornemann MA, Mahowald MW. Sleep Forensics. In: Kryger M, Roth T, Dement WC, editors. Principles and practice of sleep medicine. 5th ed. Philadelphia: W. B. Saunders; 2010.

12. Januszko P, Niemcewicz S, Gajda T, Wolynczyk-Gmaj D, Piotrowska AJ, Gmaj B, et al. Sleepwalking episodes are preceded by arousal-related activation in the cingulate motor area: EEG current density imaging. Clin Neurophysiol: Off J Int Fed Clin Neurophysiol. 2016;127(1):530-6. doi:10.1016/j.clinph.2015.01. 014.

13. Naylor MW, Aldrich MS. The distribution of confusional arousals across sleep stages and time of night in children and adolescents with sleep terrors. Sleep Res. 1991;20:308.

14. Fois C, Wright MA, Sechi G, Walker MC, Eriksson SH. The utility of polysomnography for the diagnosis of NREM parasomnias: an observational study over 4 years of clinical practice. J Neurol. 2015;262(2):385-93. doi:10.1007/s00415-014-7578-2.

15. Bjorvatn B, Gronli J, Pallesen S. Prevalence of different parasomnias in the general population. Sleep Med. 2010;11(10): 1031-4. doi:10.1016/j.sleep.2010.07.011.

16. Ohayon MM, Priest RG, Zulley J, Smirne S. The place of confusional arousals in sleep and mental disorders: findings in a general population sample of 13,057 subjects. J Nerv Ment Dis. 2000;188(6):340-8.

17. Ohayon MM, Guilleminault C, Priest RG. Night terrors, sleepwalking, and confusional arousals in the general population: their frequency and relationship to other sleep and mental disorders. J Clin Psychiatry. 1999;60(4):268-76. quiz 77.

18. Bjorvatn B, Mageroy N, Moen BE, Pallesen S, Waage S. Parasomnias are more frequent in shift workers than in day workers. Chronobiol Int. 2015;32(10):1352-8. doi:10.3109/ 07420528.2015 .1091354

19. Ingravallo F, Poli F, Gilmore EV, Pizza F, Vignatelli L, Schenck CH, et al. Sleep-related violence and sexual behavior in sleep: a systematic review of medical-legal case reports. J Clin Sleep Med: JCSM: Off Publ Am Acad Sleep Med. 2014;10(8):927-35. doi:10.5664/ jcsm.3976. This reference provides an updated review of medical-legal parasomnia case reports and proposes a framework for forensic assessment of future cases.

20. Lopez R, Jaussent I, Dauvilliers Y. Pain in sleepwalking: a clinical enigma. Sleep. 2015;38(11):1693-8. doi:10.5665/sleep.5144.

21. Lopez R, Jaussent I, Scholz S, Bayard S, Montplaisir J, Dauvilliers Y. Functional impairment in adult sleepwalkers: a case-control study. Sleep. 2013;36(3):345-51. doi:10.5665/sleep.2446.

22. Petit D, Pennestri MH, Paquet J, Desautels A, Zadra A, Vitaro F, et al. Childhood sleepwalking and sleep terrors: a longitudinal study of prevalence and familial aggregation. JAMA Pediatr. 2015;169(7):653-8. doi:10.1001/jamapediatrics.2015.127.

23. Hublin C, Kaprio J, Partinen M, Heikkila K, Koskenvuo M. Prevalence and genetics of sleepwalking: a population-based twin study. Neurology. 1997;48(1):177-81.

24. Dang-Vu TT, Zadra A, Labelle MA, Petit D, Soucy JP, Montplaisir J. Sleep deprivation reveals altered brain perfusion patterns in somnambulism. PLoS One. 2015;10(8), e0133474. doi:10.1371/ journal.pone. 0133474 .

25. Perrault R, Carrier J, Desautels A, Montplaisir J, Zadra A. Slow wave activity and slow oscillations in sleepwalkers and controls: effects of 38 h of sleep deprivation. J Sleep Res. 2013;22(4):430-3. doi:10.1111/jsr.12041.

26. Perrault R, Carrier J, Desautels A, Montplaisir J, Zadra A. Electroencephalographic slow waves prior to sleepwalking episodes. Sleep Med. 2014;15(12):1468-72. doi:10.1016/j.sleep. 2014.07.020.

27. Allison KC, Lundgren JD, O'Reardon JP, Geliebter A, Gluck ME, Vinai $\mathrm{P}$, et al. Proposed diagnostic criteria for night eating syndrome. Int J Eating Disord. 2010;43(3):241-7. doi:10.1002/eat. 20693.

28. Chen YW, Tseng PT, Wu CK, Chen CC. Zaleplon-induced anemsic somnambulism with eating behaviors under once dose. Acta Neurol Taiwanica. 2014;23(4):143-5.

29. Hoque R, Chesson Jr AL. Zolpidem-induced sleepwalking, sleep related eating disorder, and sleep-driving: fluorine-18flourodeoxyglucose positron emission tomography analysis, and a literature review of other unexpected clinical effects of zolpidem. J Clin Sleep Med: JCSM: Off Publ Am Acad Sleep Med. 2009;5(5): 471-6.

30. Nzwalo H, Ferreira L, Peralta R, Bentes C. Sleep-related eating disorder secondary to zolpidem. BMJ Case Rep. 2013. doi:10. 1136/bcr-2012-008003.

31. Paquet V, Strul J, Servais L, Pelc I, Fossion P. Sleep-related eating disorder induced by olanzapine. J Clin Psychiatry. 2002;63(7):597.

32. Tamanna S, Ullah MI, Pope CR, Holloman G, Koch CA. Quetiapine-induced sleep-related eating disorder-like behavior: a case series. J Med Case Rep. 2012;6:380. doi:10.1186/17521947-6-380.

33. Brion A, Flamand M, Oudiette D, Voillery D, Golmard JL, Arnulf I. Sleep-related eating disorder versus sleepwalking: a controlled study. Sleep Med. 2012;13(8):1094-101. doi:10.1016/j.sleep. 2012.06.012.

34. Howell MJ, Schenck CH. Restless nocturnal eating: a common feature of Willis-Ekbom syndrome (RLS). J Clin Sleep Med: JCSM: Off Publ Am Acad Sleep Med. 2012;8(4):413-9. doi:10. 5664/jcsm. 2036.

35. Antelmi E, Vinai P, Pizza F, Marcatelli M, Speciale M, Provini F. Nocturnal eating is part of the clinical spectrum of restless legs syndrome and an underestimated risk factor for increased body mass index. Sleep Med. 2014;15(2):168-72. doi:10.1016/j.sleep. 2013.08.796.

36. Sharpless BA, Barber JP. Lifetime prevalence rates of sleep paralysis: a systematic review. Sleep Med Rev. 2011;15(5):311-5. doi: 10.1016/j.smrv.2011.01.007.

37. Bell CC, Dixie-Bell DD, Thompson B. Further studies on the prevalence of isolated sleep paralysis in black subjects. J Natl Med Assoc. 1986;78(7):649-59.

38. Nadorff MR, Lambdin KK, Germain A. Pharmacological and nonpharmacological treatments for nightmare disorder. Int Rev Psychiatry (Abingdon, England). 2014;26(2):225-36. doi:10. 3109/09540261.2014.888989.

39. Berry RB, Brooks R, Gamaldo CE, Harding SM, Lloyd RM, Marcus CL, et al. The AASM manual for the scoring of sleep and associated events: rules, terminology and technical specifications, Version 2.2. Darien: American Academy of Sleep Medicine; 2015. 
40. Frauscher B, Gschliesser V, Brandauer E, Marti I, Furtner MT, Ulmer H, et al. REM sleep behavior disorder in 703 sleepdisorder patients: the importance of eliciting a comprehensive sleep history. Sleep Med. 2010;11(2):167-71. doi:10.1016/j.sleep.2009. 03.011.

41. Schenck CH, Boeve BF, Mahowald MW. Delayed emergence of a parkinsonian disorder or dementia in $81 \%$ of older men initially diagnosed with idiopathic rapid eye movement sleep behavior disorder: a 16-year update on a previously reported series. Sleep Med. 2013;14(8):744-8. doi:10.1016/j.sleep.2012.10.009.

42. Gagnon JF, Postuma RB, Montplaisir J. Update on the pharmacology of REM sleep behavior disorder. Neurology. 2006;67(5):7427. doi:10.1212/01.wnl.0000233926.47469.73.

43. Lam SP, Fong SY, Ho CK, Yu MW, Wing YK. Parasomnia among psychiatric outpatients: a clinical, epidemiologic, cross-sectional study. J Clin Psychiatry. 2008;69(9):1374-82.

44. Ju YE, Larson-Prior L, Duntley S. Changing demographics in REM sleep behavior disorder: possible effect of autoimmunity and antidepressants. Sleep Med. 2011;12(3):278-83. doi:10.1016/j.sleep. 2010.07.022.

45. Teman PT, Tippmann-Peikert M, Silber MH, Slocumb NL, Auger RR. Idiopathic rapid-eye-movement sleep disorder: associations with antidepressants, psychiatric diagnoses, and other factors, in relation to age of onset. Sleep Med. 2009;10(1):60-5. doi:10. 1016/j.sleep.2007.11.019.

46. Frauscher B, Iranzo A, Hogl B, Casanova-Molla J, Salamero M, Gschliesser V, et al. Quantification of electromyographic activity during REM sleep in multiple muscles in REM sleep behavior disorder. Sleep. 2008;31(5):724-31.

47. Frauscher B, Iranzo A, Gaig C, Gschliesser V, Guaita M, Raffelseder V, et al. Normative EMG values during REM sleep for the diagnosis of REM sleep behavior disorder. Sleep. 2012;35(6):835-47. doi:10.5665/sleep.1886.

48. Stefani A, Gabelia D, Mitterling T, Poewe W, Hogl B, Frauscher B. A prospective video-polysomnographic analysis of movements during physiological sleep in 100 healthy sleepers. Sleep. 2015;38(9): 1479-87. doi:10.5665/sleep.4994. Filling a gap in the spectrum of sleep-related movement, this study presents videopolysomnographic quantification of normal NREM and REM-related movement in healthy sleeper controls. This knowledge provides a framework for standardizing classification of movement in parasomnias and sleep-related movement disorders

49. Frandsen R, Nikolic M, Zoetmulder M, Kempfner L, Jennum P. Analysis of automated quantification of motor activity in REM sleep behaviour disorder. J Sleep Res. 2015;24(5):583-90. doi:10. 1111/jsr. 12304.

50. Frauscher B, Gabelia D, Biermayr M, Stefani A, Hackner H, Mitterling T, et al. Validation of an integrated software for the detection of rapid eye movement sleep behavior disorder. Sleep. 2014;37(10):1663-71. doi:10.5665/sleep.4076.

51. McCarter SJ, St Louis EK, Duwell EJ, Timm PC, Sandness DJ, Boeve BF, et al. Diagnostic thresholds for quantitative REM sleep phasic burst duration, phasic and tonic muscle activity, and REM atonia index in REM sleep behavior disorder with and without comorbid obstructive sleep apnea. Sleep. 2014;37(10):1649-62. doi:10.5665/sleep.4074.

52. McCarter SJ, St Louis EK, Sandness DJ, Arndt K, Erickson M, Tabatabai G, et al. Antidepressants increase REM sleep muscle tone in patients with and without REM sleep behavior disorder. Sleep. 2015;38(6):907-17. doi:10.5665/sleep.4738. Antidepressantassociated RBD and idiopathic RBD share common phenotypes but are thought not to share the same prognostic significance. This study highlights the quantifiable differences between the two subtypes during polysomnography.

53. Lee K, Baron K, Soca R, Attarian H. The prevalence and characteristics of REM sleep without atonia (RSWA) in patients taking antidepressants. J Clin Sleep Med: JCSM: Off Publ Am Acad Sleep Med. 2015.

54. Goadsby PJ, Sharpless BA. Exploding head syndrome, snapping of the brain or episodic cranial sensory shock? J Neurol Neurosurg Psychiatry. 2016. doi:10.1136/jnnp-2015-312617.

55. Frese A, Summ O, Evers S. Exploding head syndrome: six new cases and review of the literature. Cephalalgia: Int J Headache. 2014;34(10):823-7. doi:10.1177/0333102414536059.

56. Pearce JM. Clinical features of the exploding head syndrome. J Neurol Neurosurg Psychiatry. 1989;52(7):907-10.

57. Iranzo A, Santamaria J. Severe obstructive sleep apnea/hypopnea mimicking REM sleep behavior disorder. Sleep. 2005;28(2):203-6.

58. Schenck CH, Arnulf I, Mahowald MW. Sleep and sex: what can go wrong? A review of the literature on sleep related disorders and abnormal sexual behaviors and experiences. Sleep. 2007;30(6): 683-702.

59. Pilon M, Montplaisir J, Zadra A. Precipitating factors of somnambulism Impact of sleep deprivation and forced arousals. Neurology. 2008;70(24):2284-90.

60. Denis D, French CC, Rowe R, Zavos HM, Nolan PM, Parsons MJ, et al. A twin and molecular genetics study of sleep paralysis and associated factors. J Sleep Res. 2015;24(4):438-46. doi:10.1111/jsr. 12282.

61. Poceta JS. Zolpidem ingestion, automatisms, and sleep driving: a clinical and legal case series. J Clin Sleep Med: JCSM: Off Publ Am Acad Sleep Med. 2011;7(6):632-8. doi:10.5664/jcsm.1468.

62. Chen CS, Huang MF, Hwang TJ, Chen ST, Ko CH, Yen CN, et al. Clinical correlates of zolpidem-associated complex sleep-related behaviors: age effect. J Clin Psychiatry. 2014;75(11):e1314-8. doi:10.4088/JCP.13m08901.

63. Sutton EL. Profile of suvorexant in the management of insomnia Drug Des, Dev Ther. 2015;9:6035-42. doi:10.2147/dddt.s73224.

64. Schenck CH, Montplaisir JY, Frauscher B, Hogl B, Gagnon JF, Postuma R, et al. Rapid eye movement sleep behavior disorder: devising controlled active treatment studies for symptomatic and neuroprotective therapy-a consensus statement from the international rapid eye movement sleep behavior disorder study group. Sleep Med. 2013;14(8):795-806. doi:10.1016/j.sleep.2013.02.016. 